\title{
Factores motivantes en la selección de tú explícito/tácito en el español mexicano de Atlanta, EE.UU
}

\section{Motivating factors in the selection of expressed/unexpressed tu in Mexican Spanish in Atlanta, USA}

\author{
PHILIP P. LIMERICK ${ }^{\mathrm{a}}$ \\ ${ }^{a}$ Eastern Kentucky University, Richmond, KY, USA. \\ Correo electrónico: philip.limerick@eku.edu
}

\begin{abstract}
Se emplean datos de un corpus conversacional del español mexicano en Atlanta, Georgia para examinar ejemplos de la expresión del sujeto pronominal (ESP) de segunda persona singular ( $\mathrm{n}=$ 478) en términos de frecuencia y restricciones, incorporando factores tales como tiempo-modoaspecto (TMA), cambio de referencia y especificidad en un análisis de regresión logística. Los resultados sugieren que tú, al igual que otros sujetos, está fuertemente afectado por el cambio de referencia y TMA. Sin embargo, el efecto de TMA es único en el sentido de que se demuestra que el aspecto imperfecto desfavorece tú explícito en relación con otros TMA, divergiendo de estudios previos. Además, la especificidad —un factor que se ha encontrado repetidamente significativo en la bibliografía - es inoperante, lo que sugiere que tú se comporta de manera diferente en este dialecto con respecto a su sensibilidad a factores lingüísticos.
\end{abstract}

Palabras claves: segunda persona singular, expresión del sujeto pronominal, español mexicano, variación morfosintáctica.

Conversational corpus data from Mexican Spanish in Atlanta, Georgia are employed to examine tokens of second-person singular SPE $(n=478)$ in terms of frequency and constraints, incorporating factors such as TMA, switch reference, and specificity in logistic regression analyses. Results suggest that $t u$, like other subjects, is strongly impacted by switch reference and tense-mood-aspect (TMA). However, the TMA effect is unique in that imperfect aspect is shown to disfavor overt tú relative to other TMAs, diverging from previous studies. Furthermore, specificity $-\mathrm{a}$ factor found to be repeatedly significant in the literature- is inoperative, suggesting that tú behaves differently in this dialect regarding its sensitivity to linguistic factors.

Key words: second-person singular, subject pronoun expression, Mexican Spanish, morphosyntactic variation. 


\section{INTRODUCCIÓN}

La expresión del sujeto pronominal (ESP) en español, descrita como una "showcase variable in variationist sociolinguistics" (Bayley et al. 2012: 50) ha sido estudiada en numerosos análisis sociolingüísticos (ej. Cameron 1994; Travis 2005; Orozco y Guy 2008; Orozco 2015; Alfaraz 2015; Lastra y Martín Butragueño 2015; Silva-Corvalán 1994; Flores-Ferrán 2004; Travis 2007; Shin y Otheguy 2009; Carvalho y Child 2011; Otheguy y Zentella 2012; Michnowicz 2015; Torres Cacoullos y Travis 2015; Geeslin, Linford y Fafulas 2015; Geeslin y Gudmestad 2016). Los estudiosos variacionistas se han interesado principalmente por entender las frecuencias de los sujetos pronominales (SP) así como las restricciones lingüísticas y sociales que rigen la variación entre pronombres de sujeto tácitos y explícitos, como se ve en (1).

(1) si tú (explícito) lo conoces, de pies a cabeza, $\varnothing$ (tácito) no necesitas vivir veinte años, o tres años... [H32 $]^{1}$

La mayoría de las investigaciones sobre la ESP considera todas las personas gramaticales en el mismo análisis (véase las importantes excepciones abajo), no obstante, ocultando patrones para la persona individual. Puesto que las distintas personas gramaticales de los sujetos pronominales responden a diferentes factores contextuales e interaccionales (Travis 2005), es importante seguir el estudio de personas/números individuales que, hasta ahora, se ha llevado a cabo más extensamente para los sujetos de primera persona de singular (ej., Travis 2005, 2007; Travis y Torres Cacoullos 2012, de Prada Pérez 2015, Bessett 2018) y, a un menor grado, la tercera persona singular (Shin 2014; Martínez-Lara, En prensa), la tercera persona plural (Lapidus y Otheguy 2005) y la primera persona plural (Posio 2012; Limerick, En prensa).

No obstante, la segunda persona singular (tú) no ha recibido tanta atención en la bibliografía variacionista, específicamente con respecto al estudio exclusivo de una sola persona gramatical (véase, ej., Cameron 1993; Orozco 2019 para interesantes investigaciones sobre el uso de tú específico versus tú no específico). El presente trabajo pretende llenar este vacío y entender mejor los patrones de selección de la segunda persona singular, en particular los factores lingüísticos que rigen su variación para la expresión del sujeto en relación a otras personas y números gramaticales. Este análisis está guiado por las siguientes preguntas de investigación:

1. ¿Cuáles factores lingüísticos influyen en tú variable? ¿Responde tú a los mismos factores documentados en los estudios previos de ESP?

2. ¿Es consistente la dirección de efecto para tú con la de las demás personas gramaticales?

\footnotetext{
${ }^{1}$ Los códigos de los hablantes indican el sexo y la edad.
} 
Para contestar estas preguntas, se emplean los datos obtenidos de entrevistas sociolingüísticas del español mexicano hablado en Georgia (Corpus of Spanish in Georgia, Limerick 2021a) para analizar la ESP de segunda persona singular. El artículo se estructura de la siguiente manera: La sección 2 aborda la bibliografía sociolingüística previa sobre la ESP con un enfoque en las variables lingüísticas relevantes al presente estudio. Sección 3 describe la metodología utilizada para analizar la ESP y sección 4 presenta los resultados y análisis. Por último, sección 5 provee una discusión de los hallazgos y sección 6 concluye el artículo con un resumen así como algunas sugerencias para las investigaciones futuras.

\section{LAS INVESTIGACIONES PREVIAS}

Las investigaciones previas sobre la ESP variable han examinado tanto las tasas de ocurrencia como las restricciones en la alternancia entre sujetos tácitos y explícitos. En términos de la frecuencia, los estudiosos han observado una amplia gama de tasas generales de pronombres explícitos en varios dialectos de español, fluctuando desde tasas relativamente bajas en México y España $(-20 \%)$ hasta tasas mucho más altas en el caribeño $(-50 \%)$ (véase Otheguy y Zentella 2012). Con respecto a los factores que influyen en la variación de ESP, numerosas variables morfosintácticas y semánticas/pragmáticas han demostrado un impacto significativo. La Tabla 1 muestra algunos ejemplos de estas variables, los contextos particulares que favorecen el uso de SP explícitos y algunos ejemplos de estudios.

Tabla 1. Resumen de los hallazgos generales para las restricciones lingüísticas en la ESP

\begin{tabular}{|l|l|l|}
\hline \multicolumn{1}{|c|}{ Variable } & SP explícitos favorecidos & \multicolumn{1}{c|}{ Estudios } \\
\hline Persona/número & Verbos en singular & $\begin{array}{l}\text { Abreu, 2012 (Florida/Puerto Rico) } \\
\text { Bentivoglio, 1987 (Caracas) } \\
\text { Carvalho y Child, 2011 (Rivera, Uruguay) } \\
\text { Flores-Ferrán, 2004 (NYC) } \\
\text { Otheguy y Zentella, 2012 (NYC) }\end{array}$ \\
\hline $\begin{array}{l}\text { Referencia } \\
\text { ("cambio de referencia") }\end{array}$ & Cambio de referencia & $\begin{array}{l}\text { Bayley y Pease-Alvarez, 1997 (California) } \\
\text { Cameron, 1994, 1995 (San Juan/Madrid) } \\
\text { Travis, 2005 (Colombia) }\end{array}$ \\
\hline $\begin{array}{l}\text { Tiempo-Modo-Aspecto } \\
\text { (TMA) }\end{array}$ & Imperfecto, condicional & $\begin{array}{l}\text { Carvalho y Bessett, 2015 (Rivera, Uruguay) } \\
\text { Silva-Corvalán, 1982 (Los Ángeles) } \\
\text { Travis, 2007 (Colombia/Nuevo México) }\end{array}$ \\
\hline
\end{tabular}




\begin{tabular}{|c|c|c|}
\hline $\begin{array}{l}\text { Ambigüedad } \\
\text { morfológica }\end{array}$ & Formas ambiguas & $\begin{array}{l}\text { Erker y Guy, } 2012 \text { (NYC) } \\
\text { Prada Pérez, } 2009 \text { (Minorca) } \\
\text { Lastra y Martín Butragueño, } 2015 \text { (Ciudad } \\
\text { de México) } \\
\text { Michnowicz. } 2015 \text { (Yucatán) }\end{array}$ \\
\hline Clase verbal & Verbos psicológicos & $\begin{array}{l}\text { Bentivoglio, } 1987 \text { (Caracas) } \\
\text { Silva-Corvalán, } 1994 \text { (Los Ángeles) } \\
\text { Travis, } 2007 \text { (Colombia/ Nuevo México) }\end{array}$ \\
\hline Modo verbal & Indicativo & $\begin{array}{l}\text { Lastra y Martín Butragueño, } 2015 \text { (Ciudad } \\
\text { de México) }\end{array}$ \\
\hline Especificidad & $\begin{array}{l}\text { México, España: } \\
\text { Referencia específica } \\
\text { Puerto Rico: } \text { Referencia } \\
\text { no específica }\end{array}$ & $\begin{array}{l}\text { Michnowicz, } 2015 \text { (Yucatán), Cameron, } \\
1992 \text { (Madrid) } \\
\text { Cameron, } 1992 \text { (San Juan) }\end{array}$ \\
\hline Tipo de cláusula & Cláusulas principales & $\begin{array}{l}\text { Orozco y Guy, } 2008 \text { (Colombia) } \\
\text { Otheguy y Zentella, } 2012 \text { (NYC) } \\
\text { Shin y Montes-Alcalá, } 2014 \text { (NYC) }\end{array}$ \\
\hline Reflexividad & Verbos no reflexivos & $\begin{array}{l}\text { Carvalho y Child, } 2011 \text { (Rivera, Uruguay); } \\
\text { Otheguy, Zentella y Livert, } 2007 \text { (NYC) }\end{array}$ \\
\hline Priming & SP explícito previo & $\begin{array}{l}\text { Cameron, } 1994 \text { (San Juan/Madrid) } \\
\text { Flores-Ferrán, } 2002 \text { (NYC) } \\
\text { Travis, } 2005 \text { (Colombia) }\end{array}$ \\
\hline
\end{tabular}

Los factores sociales, tales como el género y la edad, aunque menos estudiados, también han demostrado tener un impacto en la ESP variable. Los hallazgos más consistentes han sido que las mujeres favorecen los SP explícitos (ej., Bayley y Pease-Alvarez 1996; Solomon 1999; Carvalho y Child 2011; Otheguy y Zentella 2012; Shin y Otheguy 2013; Alfaraz 2015) y que los hablantes más jóvenes favorecen los SP tácitos (ej., Carvalho y Child 2011; Orozco y Guy 2008; Lastra y Martín Butragueño 2015; Limerick 2019). Además, factores como la duración de la residencia, la edad de llegada, la generación de inmigrantes y el nivel de bilingüismo influyen en el uso de SP en las variedades de español en contacto con otras lenguas (véase ej., Otheguy y Zentella 2012; Carvalho, Orozco y Shin 2015).

El presente estudio se centrará principalmente en tres de estos factores: TMA, cambio de referencia y especificidad. ${ }^{2}$ La siguiente sección discutirá su influencia con

\footnotetext{
${ }^{2}$ Puede surgir una pregunta sobre la posible influencia del contacto lingüístico/bilingüismo en las tasas de SP explícito observadas en los datos actuales en comparación con las variedades mexicanas monolingües. No obstante, investigaciones previas de este mismo grupo de hablantes han descartado esta posibilidad (Limerick 2018, 2019). En particular, no se encontraron correlaciones significativas entre las tasas de pronombres explícitos y el dominio del inglés, la duración de la residencia en los EE.UU. o la edad de llegada a los EE. UU.
} 
mayor detalle de acuerdo con los hallazgos de investigaciones anteriores. Empezaremos con el factor que restringe más fuertemente a la ESP en todos los dialectos, persona/número gramatical. Aunque este factor no se emplea como variable independiente en el presente análisis (aquí se examina sola una persona/número), ayudará a establecer un fondo para la investigación de ESP.

\subsection{Personalnúmero}

En todas las variedades del español estudiadas, tanto la persona como el número del verbo influyen en gran medida en la variación de SP. De hecho, se ha demostrado que la persona/número es el predictor más fuerte de la ESP variable en todos los dialectos (Orozco 2015). Específicamente, los verbos en primera persona del singular y en tercera persona del singular tienden a favorecer los pronombres explícitos (Silva-Corvalán 1994; Flores-Ferrán 2002; Shin 2012; Lastra y Martín Butragueño 2015; Limerick 2019). De hecho, la mayoría de los estudios ha encontrado que todas las formas singulares en general tienen mayores probabilidades de aparecer con SP explícitos en comparación con las formas plurales, las cuales tienden a favorecer los SP tácitos (Orozco 2015). Además, a veces se han observado diferencias regionales. Por ejemplo, Otheguy, Zentella y Livert (2007) encontraron que los recién llegados caribeños a la ciudad de Nueva York preferían los SP explícitos con verbos en segunda persona del singular en comparación con los verbos en tercera persona del singular, pero que los recién llegados sudamericanos preferían los SP explícitos con verbos en tercera persona del singular. Cameron (1993) ha explicado el hallazgo general de personas/números singulares y plurales y su influencia en la expresión del sujeto de la siguiente manera:

If we conceive of plural subjects as sets, we find that discourse is typically structured so that the great majority of plural subjects occur in contexts where their set members are either explicitly or inferably present within the immediately preceding discourse. Such contexts favor null subject expression. Therefore, plural subjects are frequently null overall (328, nota 2$)$.

Además, la distinción más amplia que se encuentra generalmente para persona/ número es que las formas de primera persona del singular $(y o)$ favorecen más a los SP explícitos (como se indicó anteriormente) mientras que las formas de primera persona del plural (nosotros) desfavorecen fuertemente a los SP explícitos. Respecto al primer patrón, el uso alto de yo se ha atribuido a la "naturaleza egocéntrica del discurso" (Posio 2011: 795, traducción mía). Del mismo modo, como explica Morales (1986), al hacer referencia explícita a uno/a mismo/a, se satisface la necesidad pragmática del hablante de estar explícitamente presente en la conversación. El uso de yo también marca subjetividad alta (Serrano 2014), señala la posesión del turno dialógico y se influye por la naturaleza intersubjetiva de la conversación (Scheibman 2007; Serrano 2014). Con respecto a este último patrón, podría ser el caso de que nosotros rara vez se expresa debido a su longitud comparativamente más larga, así 
como al hecho de que su inflexión verbal es la menos ambigua (Bentivoglio 1987) y la más inclusiva de las personas dialógicas. De manera similar, Orozco y Guy (2008) sugieren que dado que tiene las formas verbales más morfológicamente distintas y regulares, el uso de nosotros sería redundante. Esto es particularmente convincente dado que la inflexión verbal de la primera persona del plural (-mos) se mantiene igual para todos los TMA en comparación con las formas de la primera y tercera persona del singular que son indistintas (es decir, morfológicamente ambiguas) en el imperfecto, subjuntivo y condicional.

\subsection{Cambio de referencia}

El cambio de referencia, un fenómeno que considera la continuidad versus el cambio de referencia de un sujeto a otro, también ha demostrado una influencia fuerte en la manifestación de SP en todos los dialectos estudiados (ej., Bentivoglio 1987; Cameron 1994; Silva-Corvalán 1994; Bayley y Pease-Alvarez 1997; Travis 2005; Prada Pérez 2009; Torres Cacoullos y Travis 2010; Carvalho y Child 2011; Otheguy y Zentella 2012; Michnowicz 2015; Orozco 2015). En concreto, en los contextos donde hay un cambio de referente, el SP a menudo es explícito, como se ve con tú en (2); cuando no hay un cambio (el sujeto se mantiene), SP tácitos son preferidos, como en (3).

(2) no están esperando a que tú llegues [M28]

(3) ...combinación de provincia y ciudad en dónde vas a encontrar diversidad en donde $\varnothing$ vas a encontrar este... [M39]

Se piensa que dicho patrón generalmente tiene una influencia funcional que tiene que ver con el seguimiento de los referentes (Shin y Otheguy 2009). Como explica Cameron (1994: 40-41), "expressed pronominal subjects compensate for the change of information state which occurs with a switch in subject reference". En otros términos, los SP explícitos tienden a usarse en tales contextos para facilitar la interpretación del antecedente para el oyente. Según Shin y Otheguy (2009), esto es especialmente importante para los referentes de tercera persona con el fin de desambiguar entre él y ella, como se ilustra en el siguiente ejemplo de sus datos:

(4) Ella tenía su novio allá y él pensaba venir pero no le dieron la visa (p. 120, énfasis mía)

Estos autores arguyen que dado que existen referentes en competencia en (4) (ella y su novio), el uso de él ayuda al oyente a seguir el antecedente de pensaba. Además, algunos investigadores también incluyen una categoría adicional, cambio parcial, que analiza los casos de cambio de sujeto donde el sujeto es correferencial con el objeto inmediatamente anterior (objeto directo/ indirecto, objeto de preposición, etc.), como en (5).

(5) ...en México me inculcó mi mamá que tenía que ir a la iglesia... [H52] 
En este ejemplo, hay un cambio de sujeto de mi mamáa yo (tenía), y yo es correferencial con el objeto anterior me. En términos generales, los estudios previos han reportado un leve favorecimiento de los SP explícitos, o un efecto neutral para tales contextos, es decir, ni un efecto favorable ni desfavorable (ej., Orozco y Guy 2008; Otheguy y Zentella 2012; Lastra y Martín Butragueño 2015; Orozco 2015).

\subsection{Tiempo-modo-aspecto (TMA)}

También se ha demostrado que el tiempo-modo-aspecto (TMA) de un verbo condiciona los SP. Ciertos TMA favorecen los SP explícitos mientras que otros TMA favorecen los tácitos. Por ejemplo, Silva Corvalán (1982) encontró que los imperfectos y los condicionales favorecen los SP explícitos mientras que los presentes y los pretéritos son más propensos a aparecer con tácitos, como también encontraron otros investigadores (ej., Cameron 1994; Travis 2007; Carvalho y Bessett 2015). Para explicar tales correlaciones, se ha propuesto que los imperfectos y los condicionales favorecen a los SP explícitos debido a su potencial ambigüedad. Dado que sus formas verbales de primera y tercera persona del singular son morfológicamente indistintas, el uso de SP explícitos serviría para desambiguar los referentes de tales formas, una explicación que forma parte de la Hipótesis Funcional (Hochberg 1986). Sin embargo, otros estudios no han encontrado tal correlación (ej., Enríquez 1984; Bentivoglio 1987; Ranson 1991).

Silva-Corvalán (2001) ha propuesto una explicación alternativa para el efecto TMA, que no está relacionada con la ambigüedad referencial. Ella discute los imperfectos y los pretéritos en relación con las funciones del discurso, sugiriendo que se utilizan más SP explícitos con los imperfectos debido a la naturaleza de fondo (menos prominentes o en segundo plano) del aspecto imperfecto, y que se utilizan menos SP explícitos con los pretéritos, ya que tienden a poner en primer plano los eventos. Es decir, para eventos que están más en el fondo, el enfoque está en el sujeto (por lo tanto, el uso de un SP explícito) mientras que el enfoque está más en la acción con más eventos en primer plano (de ahí la falta de un SP explícito). Sin embargo, esta hipótesis ha sido rechazada en trabajos más recientes. Por ejemplo, Shin (2014) arguye más bien por la explicación de la ambigüedad, encontrando que los imperfectos favorecieron a los SP explícitos, particularmente en contextos de cambio de referencia y con referentes en competencia (donde el contexto presenta dos o más referentes posibles para un verbo en el imperfecto), ambos contextos en los que el seguimiento de referentes es más difícil. Dados sus hallazgos, Shin sostiene que su análisis no apoya la propuesta de Silva-Corvalán (2001).

\subsection{Especificidad}

El uso de un referente específico comparado a un referente más general (por ejemplo, tú impersonal), también tiende a condicionar si un pronombre es explícito o tácito. Las investigaciones anteriores han demostrado que el efecto de la especificidad sobre la ESP puede depender del dialecto. Por ejemplo, las obras de Cameron $(1992,1993)$ 
sobre el español de San Juan y Madrid demuestra que los referentes de sujeto no específicos favorecieron a los SP explícitos en San Juan mientras que los referentes específicos favorecieron a los SP explícitos en Madrid. En cuanto al español mexicano, tanto Solomon (1999) como Michnowicz (2015) encontraron que, al igual que el español de Madrid, los SP explícitos fueron favorecidos por referentes específicos.

\section{Metodología}

\subsection{Recopilación de datos}

En 2015 conduje entrevistas sociolingüísticas con hispanohablantes quienes vivían en Roswell, Georgia, un suburbio de Atlanta, en el tiempo de la recopilación de datos. La muestra para el presente análisis consiste en 20 inmigrantes mexicanos de primera generación. Estas entrevistas forman parte del Corpus of Spanish in Georgia (Limerick 2021a). Las entrevistas duraron entre 30 minutos y una hora. Eran informales, conversacionales y abordaron temas tales como la historia personal, la vida en la comunidad local, las diferencias entre los países natales de los hablantes y los Estados Unidos y las experiencias integrándose a la vida en Roswell, entre otros temas.

\subsection{Los hablantes}

Los antecedentes sociodemográficos de los hablantes se pueden resumir de la siguiente manera: Los hablantes nacieron en varias regiones de México: Ciudad de México (8), Acapulco, Guerrero (2), el estado de Guerrero (1), Juando, México (1), el estado de Zacatecas (1), Cuernavaca, Morelos (1), el estado de Morelos (1), Tampico, Tamaulipas (1), San Juan del Río, Querétaro (1), Monterrey, Nuevo León (1), el estado de Colima (1) y el estado de San Luis Potosí (1). Constan de 12 mujeres y 8 hombres, y sus edades fluctúan entre los 25 y los 60 ańos. Además, sus duraciones de residencia en los EE.UU. varían de 2 a 25 ańos (promedio $=12$ años), y sus edades de llegada varían de 11 a 56 (promedio = 27 ). En términos de niveles educativos, van desde la escuela primaria hasta la universidad. Los hablantes tienen una variedad de ocupaciones, casi la mitad de ellos son propietarios de pequeñas empresas. Por último, su dominio del inglés varía de muy pobre a bueno. El dominio del inglés se midió utilizando las autoevaluaciones de los hablantes en una escala de 1 (muy pobre) a 5 (muy bueno), con una evaluación promedio de 2.75 .

\subsection{El contexto variable}

Para el análisis, extraje todos los verbos en segunda persona del singular de las entrevistas. Para aislar solo los casos en los que se produjo variación entre un SP explícito y tácito, he excluido los siguientes tipos de ejemplos que se quedaron fuera del contexto variable: 
a. Imperativos (ej. siempre me dijo, "Ø estudia, $\varnothing$ agarra un libro..." [M52Mex])

b. Frases fijadas con sujetos tácitos categóricos (sin ningún caso de SP explícito) (ej. $\varnothing$ ¿me entiendes?; $\varnothing$ ¿ves?).

Los hablantes no alternaron entre un SP explícito y tácito en los casos de arriba (los hablantes usaron sujetos tácitos exclusivamente); por consiguiente, estas estructuras fueron excluidas. Para todos los ejemplos dentro del contexto variable $(\mathrm{N}=478)$, primero codifiqué si cada verbo apareció con un SP tácito o explícito. Posteriormente, codifiqué las tres variables independientes utilizando las categorizaciones discutidas en las siguientes secciones.

\subsection{Variables independientes}

Los tres predictores internos para la presente investigación son los siguientes: Cambio de Referencia, TMA y Especificidad. La inclusión de estos predictores se basa en su importancia en investigaciones previas de ESP, y los métodos de codificación se basan principalmente en el análisis de Otheguy y Zentella (2012) del español de Nueva York, así como en el estudio de Lastra y Martín Butragueño (2015) sobre el español de la Ciudad de México (véase Tabla 2). Puesto que el conjunto de datos es relativamente pequeño, se limitó el número de predictores incluidos para evitar un modelo estadístico demasiado complejo, aumentando así la validez de los resultados. Estos tres factores en particular se eligieron para facilitar la comparabilidad con estudios anteriores, los cuales han reportado efectos consistentemente fuertes para los factores bajo estudio.

Tabla 2. Variables y categorías

\begin{tabular}{|l|l|}
\hline \multicolumn{1}{|c|}{ Variable } & \multicolumn{1}{c|}{ Categorías } \\
\hline Cambio de referencia & $\begin{array}{l}\text { Continuidad de referencia } \\
\text { Cambio de referencia } \\
\text { Cambio parcial (los sujetos que son correferenciales con el objeto } \\
\text { anterior), como se ve en el siguiente ejemplo: } \\
\text { Te ocupan, te utilizan, y te dicen si trabajas bien... [M43] }\end{array}$ \\
\hline TMA & $\begin{array}{l}\text { Presente de indicativo, pretérito, imperfecto, perfecto (incluido el pre- } \\
\text { sente perfecto y pluscuamperfecto), presente de subjuntivo, pasado de } \\
\text { subjuntivo, futuro sintético, futuro perifrástico, condicional } \\
\text { *No se incluyó el modo imperativo como era categóricamente tácito. }\end{array}$ \\
\hline Especificidad & $\begin{array}{l}\text { Específico } \\
\text { No específico }\end{array}$ \\
\hline
\end{tabular}




\subsection{Métodos estadísticos}

Para determinar la significancia estadística de las variables lingüísticas y el peso relativo de cada factor con respecto a su condicionamiento al uso de ESP, se realizaron análisis multivariados de efectos mixtos (regresión logística) utilizando Rbrul (Johnson 2009) con la inclusión del hablante como un efecto aleatorio. Un análisis multivariado nos permite determinar el efecto relativo de múltiples factores a la vez. Específicamente, facilita la producción de una jerarquía para los factores estadísticamente significativos basados en la cantidad de variación explicada por cada factor. Además, los resultados cuantitativos ayudan a crear una jerarquía de probabilidades dentro de un solo grupo de factores (ej., Cambio de referencia), es decir, las probabilidades dentro de un grupo de factores que favorecen a los SP explícitos (ej., cambio de referencia) versus aquellos que no favorecen a los SP explícitos (ej., continuidad de referencia). Además, la inclusión del hablante como un efecto aleatorio en el análisis multivariado es una forma de controlar por el hablante individual y asegurar de que los resultados obtenidos sean generalizables al conjunto de datos en general, y que los patrones observados no se deben a que ningún hablante en particular sesgue los resultados (Bayley, Greer y Holland 2013; Shin 2014; Michnowicz 2015; Shin y Van Buren 2016).

La siguiente sección presenta los resultados del presente análisis. Primero, sin embargo, es importante señalar algunas modificaciones metodológicas realizadas al conjunto de datos: debido al recuento bajo de ejemplos para algunas de las categorías, estas se colapsaron. Específicamente, la categoría cambio parcial se movió a la categoría de cambio, y las seis categorías de TMA de perfecto, presente de subjuntivo, pasado de subjuntivo, futuro sintético, futuro perifrástico y condicional se movieron a una categoría separada llamada Los demás TMA (ver Lastra y Martín Butragueño, 2015 para métodos similares). Por último, todos los casos del pretérito $(\mathrm{N}=11)$ fueron excluidos por ser categóricamente tácitos ${ }^{3}$.

\section{Resultados}

Con respecto a la tasa pronominal general para tú, el pronombre explícito se produjo en $14 \%$ de los casos, lo cual es consistente con los estudios previos; esta tasa es muy comparable a lo que se ha reportado para otras variedades de español mexicano (ej., Lastra y Martín Butragueño 2015; Michnowicz 2015; Shin y Van Buren 2016), como se ve en la Tabla 3.

\footnotetext{
3 Además, para explorar posibles diferencias regionales, se realizó un análisis de regresión por separado que también incluyó Región como una variable independiente (Ciudad de México vs. No Ciudad de México). La región no resultó significativa $(p=0.13)$, lo que sugiere una falta de variación según el origen regional en México.
} 
Tabla 3. Distribución de tú explícito y tácito $(\mathrm{n}=478)$

\begin{tabular}{|c|c|c|c|c|}
\hline & $\begin{array}{c}\text { Español } \\
\text { mexicano en } \\
\text { Georgia (presente } \\
\text { estudio) }\end{array}$ & $\begin{array}{c}\text { Español de D.F. } \\
\text { (Lastra y Martín } \\
\text { Butragueńo } \\
2015: 43)\end{array}$ & $\begin{array}{c}\text { Mexicanos en } \\
\text { Washington/ } \\
\text { Montana(Shin y Van } \\
\text { Buren 2016: 184) }\end{array}$ & $\begin{array}{c}\text { Español yucateco } \\
\text { (Michnowicz } \\
2015: 109)\end{array}$ \\
\hline$\%$ explícito & $14 \%$ & $17 \%$ & $17 \%$ & $15 \%$ \\
\hline$\%$ tácito & $86 \%$ & $83 \%$ & $83 \%$ & $85 \%$ \\
\hline
\end{tabular}

En cuanto a la influencia de variables lingüísticas sobre la variación de SP, el análisis multivariado demostró que dos factores resultaron estadísticamente significativos: El cambio de referente y TMA. Por el contrario, la especificidad no resultó significativa $(p=0.465)$.

La Tabla 4 abajo presenta las jerarquías de restricciones para cada factor significativo. La primera columna muestra los factores junto con sus niveles particulares, y la tercera columna presenta los pesos probabilísticos (PP) para cada restricción desde la probabilidad más alta hasta la más baja de aparecer con un SP explícito. Cuando un PP está más cercano a 1, indica una preferencia por SP explícitos. Cuando el PP está más cercano a 0, por lo general indica una preferencia por SP tácitos (veáse Tagliamonte 2006: 145, 156). A continuación discutiré los resultados de cada factor significativo como se ve en la Tabla 4.

Tabla 4. Jerarquías de restricciones para ESP de segunda persona singular $(\mathrm{n}=478)^{*}$

\begin{tabular}{|l|c|c|c|c|c|}
\hline \multicolumn{1}{|c|}{ Variable } & $\begin{array}{c}\text { Razones lógicas } \\
\text { (Logodds) }\end{array}$ & $\begin{array}{c}\text { Peso } \\
\text { probabilístico }\end{array}$ & \% explícito & N total & Valor- $\boldsymbol{p}$ \\
\hline TMA & & & & & 0.0178 \\
\hline Los demás TMA & 0.922 & .72 & $24 \%$ & 59 & \\
\hline Presente & -0.313 & .42 & $13 \%$ & 399 & \\
\hline Imperfecto & -0.609 & .35 & $5 \%$ & 20 & \\
\hline RANGO = 37 & & & & & \\
\hline Cambio de referencia & & .67 & $19 \%$ & 272 & \\
\hline Cambio & 0.708 & .33 & $7 \%$ & 206 & \\
\hline Continuidad & -0.708 & & & & \\
\hline RANGO = 34 & & & & & \\
\hline
\end{tabular}




\subsection{Cambio de referencia}

Consistente con el hallazgo repetido en estudios previos de ESP variable en general (ej., Travis 2005; Torres Cacoullos y Travis 2010; Carvalho y Child 2011; Otheguy y Zentella 2012; Michnowicz 2015; Orozco 2015), un cambio de referente del sujeto promovió el uso de tú explícito en los presentes datos ( $\mathrm{PP}=.67 ; 19 \%$ explícito) mientras que la continuidad del referente favoreció el SP tácito $(\mathrm{PP}=.33 ; 7 \%$ explícito). Aparte de ser consistente con la investigación previa por lo general, este hallazgo también ofrece apoyo al análisis de Cameron (1995), quien aseveró que el cambio de referencia es una restricción que se aplica a todas las personas gramaticales. Puesto que hay poca investigación sobre tú solo, los hallazgos aquí confirman que los hablantes siguen siendo sensibles a un cambio de referente del sujeto al utilizar la expresión del sujeto segunda persona singular. Dicho patrón se ilustra en (6), en el cual hay un cambio de es a tú entras:

(6) Pues tan fácil no es, realmente es un reto, desde que tú entras a este país es un reto [M32]

\subsection{Tiempo-modo-aspecto (TMA)}

Sorprendentemente, el patrón gramatical para TMA es bastante anómalo en comparación con la mayoría de los estudios previos. En particular, el efecto desfavorecedor de SP explícitos con el imperfecto ( $\mathrm{PP}=.35$ ) no era de esperar y contradice los hallazgos generales en la bibliografía (tanto los estudios que toman en cuenta todas las personas gramaticales como los que incluyen solo una persona) que demuestran una preferencia fuerte por SP explícitos con el imperfecto al compararse con otros TMA (Silva Corvalán 1982; Cameron 1994; Travis 2007; Carvalho y Bessett 2015; Orozco 2015, 2016; Shin y Van Buren 2016; Lastra y Martín Butragueño 2015). No obstante, el hallazgo presente es consistente con nosotros variable (Limerick, En prensa). Regresaré a estos hallazgos en la sección 5. Los ejemplos (7) y (8) abajo demuestran el uso de tú explícito con Los demás TMA (el pasado de subjuntivo en este caso) así como el sujeto tácito con el imperfecto, respectivamente.

(7) ...porque si tú vieras a mi papá pues... típico, americano... [H41]

(8) ...y pues eran, juegos en la calle... físicos, Ø salias a correr... [M26]

\section{Discusión}

En esta sección, vuelvo a las preguntas de investigación iniciales para empezar a ofrecer respuestas a estas preguntas en cuanto al comportamiento de ESP de 2 sg. Después, concluyo por resumir los hallazgos y sugerir algunas direcciones futuras para la investigación sobre la ESP variable. 


\section{1. ¿Cuáles factores lingüisticos influyen en tú variable? ¿Responde tú a los mismos factores documentados en los estudios previos de ESP?}

Para los presentes datos, tú variable está restringido por dos factores lingüísticos: El cambio de referente y TMA. Dichos factores suelen resultar significativos en estudios sobre la ESP. Sin embargo, otro factor que típicamente demuestra un efecto en estudios previos (la especificidad) no impactó el uso variable de tú de modo estadísticamente significativo en el presente trabajo. No obstante, las tasas observadas (22\% específico vs. $13 \%$ no específico; $\mathrm{PP}=.55$ y .46 , respectivamente) sí indican patrones semejantes a los de otras variedades mexicanas (Michnowicz 2015; Shin 2016). Además, la preferencia de tú explícito para referencia específica es consistente con variedades dominicanas (ej., Martínez-Sanz 2011; Alfaraz 2015) y peninsulares (Cameron 1993), no en términos de las tasas generales, pero en el sentido de que las tasas para tú específico son más altas que las tasas de tú no específico (ej., Alfaraz 2015: específico 85\% vs. no específico: 66\%). El patrón opuesto — tasas más altas para tú no específico- también ha sido observado para variedades puertorriqueñas (ej., Cameron 1993) y uruguayas (Carvalho y Bessett 2015).

\section{2. ¿Es consistente la dirección de efecto para tú con la de las demás personas gramaticales?}

En cuanto al cambio de referencia, la dirección de efecto es consistente con los hallazgos robustos en la bibliografía, a saber que un cambio de sujeto favorece el uso de SP explícitos en general. El mismo patrón se mantiene para tú en particular. Para TMA, no obstante, la dirección de efecto demuestra patrones sumamente divergentes a aquellos reportados en investigaciones previas: En lugar de favorecer los SP explícitos, los imperfectos (así como el tiempo presente) prefirieron los $\mathrm{SP}$ tácitos $(\mathrm{PP}=.35)$. Además, los demás TMA prefirieron tú explícito.

¿Por qué producirían los hablantes menos tú explícito en los contextos del imperfecto? Una razón posible está relacionada con la hipótesis funcional (Hochberg 1986). Puesto que la morfología verbal de segunda persona singular no es ambigua, no hay motivo por compensar la información de persona gramatical. Se sostiene lo mismo para el tiempo presente. Este hallazgo proporciona apoyo a favor de la hipótesis de desambiguación, pues son sólo las formas morfológicamente ambiguas (1sg y 3sg del imperfecto) que favorecen el uso de pronombres explícitos. De hecho, utilizando estos mismos datos, Limerick (2021b) encontró que el patrón típico para TMA sí se mantuvo con 1 sg y 3sg, pero no para nosotros (Limerick, En prensa). Estos hallazgos juntos, por lo tanto, dan más apoyo a la ambigüedad y menos apoyo para el efecto de primer plano/fondo de Silva-Corvalán (2001).

Otra pregunta que surge es si los hallazgos para TMA tienen que ver simplemente con la distribución de los datos. ¿Los contextos imperfectos, por ejemplo, se superponen con los contextos de continuidad de referencia en los datos, disminuyendo así la tasa de sujetos explícitos? Del mismo modo, ¿se superponen Los demás TMA con los contextos de cambio de referencia, aumentando la tasa pronominal? Una exploración de la distribución 
de datos no mostró una cantidad desproporcionada de acoplamiento entre la continuidad de referencia y contextos del imperfecto en relación con otros TMA. De hecho, la proporción de los ejemplos de continuidad de referencia para imperfecto $(25 \% ; 5 / 20)$ fue menor que para los demás TMA (37\%; 22/59), e incluso menor que la del tiempo presente (45\%; 179/399). Por lo tanto, el solapamiento potencial entre el imperfecto y continuidad de referencia que de otro modo podría explicar la tasa más baja de tú explícito con el imperfecto simplemente no se confirma en la distribución de los presentes datos. Por decirlo de otra manera, no existe el solapamiento entre los diferentes TMA y cambio de referencia que, si esto fuera así, explicaría la tasa más alta de tú para los demás TMA. Las investigaciones futuras son necesarias para determinar si este es un patrón general para el español mexicano o simplemente una característica idiosincrásica de los presentes datos.

\section{Conclusión}

Los presentes hallazgos han confirmado los estudios previos de ESP y también plantean preguntas importantes con respecto al estudio de ESP en español. Dados los hallazgos diferenciales para la segunda persona singular en relación a otras personas/ números, los presentes resultados ofrecen apoyo a los investigadores que han enfatizado la importancia de estudiar personas gramaticales individuales en vez de combinar todas las personas juntos en el mismo modelo (Travis 2005, 2007; Travis y Torres Cacoullos 2012; Shin 2014).

El trabajo futuro es necesario para descubrir las matices adicionales en la gramática variable de sujetos pronominales de los hispanohablantes. Primero, los factores lingüísticos adicionales así como factores sociales (ej., priming, frecuencia léxica, edad, género, educación) y las interacciones entre dichos factores se deben incorporar para investigar más a fondo y explicar la variación en la segunda persona singular de ESP. Segundo, más investigaciones son necesarias sobre el efecto de TMA sobre la segunda persona singular para confirmar los patrones observados en los presentes dados. Esto podría incluir, entre otras aproximaciones, un análisis cualitativo detallado que pueda potencialmente revelar los motivos para el desfavorecimiento de tú explícito con respecto al imperfecto. Además, ¿podrían los presentes hallazgos representar rasgos dialectales? ¿Qué muestran las otras variedades (mexicanas y no mexicanas) para la ESP variable en la segunda persona singular? Por último, puesto que el conjunto de datos para el presente estudio fue relativamente pequeño, los hallazgos son tentativos. Los análisis futuros se beneficiarían de tener un corpus de hablantes más grande para aumentar las posibilidades de generalización de los resultados.

En resumen, mientras tú respondió a algunos de los mismos factores reportados en la bibliografía de ESP en general (cambio de referencia), no hubo ningún efecto para la especificidad. Además, el efecto TMA divergió significativamente de las investigaciones previas, particularmente con respecto al patrón gramatical de restricciones que indicó un desfavorecimiento de tú explícito con el imperfecto. Las futuras investigaciones ayudarán 
a determinar si dichos patrones se mantienen para español en general o si son un reflejo de este dialecto de espańol mexicano en particular.

\section{Obras Citadas}

Abreu, Laurel. 2012. "Subject pronoun expression and priming effects among bilingual speakers of Puerto Rican Spanish". En Kimberly Geeslin y Manuel Díaz-Campos, Eds., Selected Proceedings of the 14th Hispanic Linguistics Symposium. Somerville, MA: Cascadilla Proceedings Project. 1-8.

Alfaraz, Gabriela G. 2015. "Variation of overt and null subject pronouns in the Spanish of Santo Domingo". En Ana M. Carvalho, Rafael Orozco y Naomi Lapidus Shin, Eds., Subject pronoun expression in Spanish: A cross-dialectal perspective. Georgetown: Georgetown University Press. 3-16.

Bayley, Robert, Kristen Greer y Cory C. Holland. 2013. "Lexical frequency and syntactic variation: A test of a linguistic hypothesis", En University of Pennsylvania Working Papers in Linguistics 19.2: 21-30.

Bayley, Robert y Lucinda Pease-Alvarez. 1996. "Null and expressed pronoun variation in Mexican-descent children's Spanish". En Jennifer Arnold, Renee Blake y Brad Davidson, Eds., Sociolinguistic Variation: Data, Theory, and Analysis. Stanford: Center for the Study of Language and Information. 85-99.

. 1997. "Null pronoun variation in Mexican-descent children's narrative discourse". Language Variation and Change 9: 349-371.

Bayley, Robert, Norma L. Cárdenas, Belinda Treviño Schouten y Carlos Martin Vélez Salas. 2012. "Spanish Dialect Contact in San Antonio, Texas: An Exploratory Study". En Kimberly Geeslin y Manuel Díaz-Campos, Eds., Selected Proceedings of the 14th Hispanic Linguistics Symposium. Somerville, MA: Cascadilla Proceedings Project. 48-60.

Bentivoglio, Paola. 1987. Los sujetos pronominales de primera persona en el habla de Caracas. Caracas: Universidad Central de Venezuela.

Bessett, Ryan M. 2018. “Testing English influence on first person singular "yo” subject pronoun expression in Sonoran Spanish". En Jonathan E. Macdonald, Ed., Contemporary Trends in Hispanic and Lusophone Linguistics: Selected papers from the Hispanic Linguistic Symposium 2015. Amsterdam: John Benjamins. 355-372.

Cameron, Richard. 1992. Pronominal and null subject variation in Spanish: Constraints, dialects, and functional compensation. Tesis doctoral, University of Pennsylvania.

1993. "Ambiguous agreement, functional compensation, and nonspecific tú in the Spanish of San Juan, Puerto Rico, and Madrid, Spain". Language Variation and Change 5: 305-34.

. 1994. "Switch reference, verb class and priming in a variable syntax" en Papers

from the regional meeting of the Chicago Linguistic Society: Parasession on variation in linguistic theory 30: 27-45. 
. 1995. "The scope and limits of switch reference as a constraint on pronominal subject expression", Hispanic Linguistics 6.7: 1-27.

Carvalho, Ana M. y Ryan M. Bessett. 2015. "Subject pronoun expression in Spanish in contact with Portuguese”. En Ana M. Carvalho, Rafael Orozco y Naomi Lapidus Shin, Eds., Subject pronoun expression in Spanish: A cross-dialectal perspective. Georgetown: Georgetown University Press. 143-65.

Carvalho, Ana M. y Michael Child. 2011. "Subject pronoun expression in a variety of Spanish in contact with Portuguese". En Jim Michnowicz y Robin Dodsworth, Eds., Selected Proceedings of the 5th Workshop on Spanish Sociolinguistics. Somerville, MA: Cascadilla Proceedings Project. 14-25.

Carvalho, Ana M., Rafael Orozco y Naomi Lapidus Shin (eds.). 2015. Subject pronoun expression in Spanish: A cross-dialectal perspective. Georgetown: Georgetown University Press.

Enríquez, Emilia V. 1984. El pronombre personal sujeto en la lengua española hablada en Madrid. Madrid: Consejo Superior de Investigaciones Científicas.

Erker, Daniel y Gregory R. Guy. 2012. "The role of lexical frequency in syntactic variability: Variable subject personal pronoun expression in Spanish”. Language 88: 526-57.

Flores-Ferrán, Nydia. 2002. Subject personal pronouns in Spanish narratives of Puerto Ricans in New York City: A sociolinguistic perspective. Munich: Lincom Europa. . 2004. "Spanish subject personal pronoun use in New York City Puerto Ricans: Can we rest the case of English contact?". Language Variation and Change 16: 49-73.

Geeslin, Kimberly y Aarnes Gudmestad. 2016. "Subject expression in Spanish: Contrasts between native and non-native speakers for first and second-person singular referents". Spanish in Context 13.1: 53-79.

Geeslin, Kimberly, Bret Linford y Stephen Fafulas. 2015. "Variable subject expression in second language Spanish: Uncovering the developmental sequence and predictive linguistic factors". En Ana M. Carvalho, Rafael Orozco y Naomi Lapidus Shin, Eds., Subject pronoun expression in Spanish: A cross-dialectal perspective. Georgetown: Georgetown University Press. 191-210.

Hochberg, Judith G. 1986. "Functional compensation for/s/ deletion in Puerto Rican Spanish". Language 62.3: 609-21.

Johnson, Daniel E. 2009. "Getting off the Goldvarb standard: Introducing Rbrul for mixed-effects variable rule analysis". Language and Linguistics Compass 3: 359-83.

Lapidus, Naomi y Ricardo Otheguy. 2005. "Overt nonspecific ellos in Spanish in New York”. Spanish in Context 2.2: 157-74.

Lastra, Yolanda y Pedro Martín Butragueño. 2015. "Subject pronoun expression in oral Mexican Spanish”. En Ana M. Carvalho, Rafael Orozco y Naomi Lapidus Shin, Eds., Subject pronoun expression in Spanish: A cross-dialectal perspective. Georgetown: Georgetown University Press. 39-57.

Limerick, Philip P. En prensa. "First-person plural subject pronoun expression in Mexican Spanish spoken in Georgia”. Studies in Hispanic and Lusophone Linguistics. 
. 2018. Subject expression in a Southeastern U.S. Mexican Community. Tesis doctoral, University of Georgia.

. 2019. "Subject expression in a Southeastern U.S. Mexican Community". Borealis

- An International Journal of Hispanic Linguistics 8.2: 243-273.

- 2021a. Corpus of Spanish in Georgia (CSG). https://encompass.eku.edu/ corpusspanga/

. 2021b. "First-person singular and third-person subject pronoun variation: The case of Mexican Spanish in the U.S. state of Georgia”. Lenguaje 49.1: 104-134.

Martínez-Lara, José Alejandro. En prensa. "Sujeto variable de $3^{a}$ persona singular en el habla de Montevideo". Onomázein.

Martinez-Sanz, Cristina. 2011. Null and overt subjects in a variable system: The case of Dominican Spanish. Tesis doctoral, University of Ottawa.

Michnowicz, Jim. 2015. "Subject pronoun expression in contact with Maya in Yucatan Spanish”. En Ana M. Carvalho, Rafael Orozco y Naomi Lapidus Shin, Eds., Subject pronoun expression in Spanish: A cross-dialectal perspective. Georgetown: Georgetown University Press. 101-119.

Morales, Amparo. 1986. Gramáticas en contacto: Análisis sintácticos sobre el español de Puerto Rico. Madrid: Playor.

Orozco, Leonor. 2019. "Tú genérico en el español de México". Borealis: An International Journal of Hispanic Linguistics 8.2: 275-294.

Orozco, Rafael y Gregory R. Guy. 2008. "El uso variable de los pronombres sujetos: ¿Qué pasa en la costa Caribe colombiana?”. En Maurice Westmoreland y Juan Antonio Thomas, Eds., Selected Proceedings of the 4th Workshop on Spanish Sociolinguistics. Somerville, MA: Cascadilla Proceedings Project. 70-80.

Orozco, Rafael. 2015. "Pronominal variation in Colombian Costeño Spanish". En Ana M. Carvalho, Rafael Orozco y Naomi Lapidus Shin, Eds., Subject pronoun expression in Spanish: A cross-dialectal perspective. Georgetown: Georgetown University Press. 17-37. . 2016. "Subject pronoun expression in Mexican Spanish: ¿Qué pasa en Xalapa?". En Proceedings of the Linguistic Society of America Vol. 1, Article 7. 1-15.

Otheguy, Ricardo y Ana Celia Zentella. 2012. Spanish in New York: Language contact, dialectal leveling, and structural continuity. Oxford University Press: New York.

Otheguy, Ricardo, Ana Celia Zentella y David Livert. 2007. "Language and dialect contact in Spanish in New York: Toward the formation of a speech community". Language 83: 770-802.

Posio, Pekka. 2011. "Spanish subject pronoun usage and verb semantics revisited: First and second person singular subject pronouns and focusing of attention in spoken Peninsular Spanish”. Journal of Pragmatics 43.3: 777-98.

2012. "Who are 'we' in spoken Peninsular Spanish and European Portuguese? Expression and reference of first person plural subjects". Language Sciences 34.3: 339-360.

Prada Pérez, Ana de. 2009. Subject expression in Minorcan Spanish: Consequences of contact with Catalan. Tesis doctoral, Pennsylvania State University. 
. 2015. "First person singular subject pronoun expression in Spanish in contact with Catalan”. En Ana M. Carvalho, Rafael Orozco y Naomi Lapidus Shin, Eds., Subject pronoun expression in Spanish: A cross-dialectal perspective. Georgetown: Georgetown University Press. 121-142.

Ranson, Diana L. 1991. "Person marking in the wake of/s/deletion in Andalusian Spanish". Language Variation and Change 3: 133-152.

Scheibman, Joanne. 2007. "Subjective and intersubjective uses of generalizations in English conversations”. En Robert Englebretson, Ed., Stancetaking in Discourse: Subjectivity, Evaluation, Interaction. Amsterdam: John Benjamins. 111-138.

Serrano, María J. 2014. "El sujeto y la subjetividad: Variación del pronombre 'yo' en géneros conversacionales y de los medios de comunicación del español de Canarias". Revista signos 47.85: 321-343.

Shin, Naomi Lapidus. 2012. "Variable use of Spanish subject pronouns by monolingual children in Mexico". En Kimberly Geeslin y Manuel Díaz-Campos, Eds., Selected Proceedings of the 14th Hispanic Linguistics Symposium. Somerville, MA: Cascadilla Proceedings Project. 130-141.

. 2014. "Grammatical complexification in Spanish in New York: 3sg pronoun expression and verbal ambiguity". Language Variation and Change 26: 303-30.

. 2016. "Children's Spanish subject pronoun expression: A developmental change in tú?". En Sandro Sessarego y Fernando Tejedo-Herrero, Eds., Spanish Language and Sociolinguistic Analysis. Amsterdam/Philadelphia: John Benjamins. 155-176.

Shin, Naomi Lapidus y Cecilia Montes-Alcalá. 2014. "El uso contextual del pronombre sujeto como factor predictivo de la influencia del inglés en el español en Nueva York". Sociolinguistic Studies 8.1: 85-110.

Shin, Naomi Lapidus y Jackelyn Van Buren. 2016. "Maintenance of Spanish subject pronoun expression patterns among bilingual children of farmworkers in Washington/Montana". Spanish in Context 13.2: 173-194.

Shin, Naomi Lapidus y Ricardo Otheguy. 2009. "Shifting sensitivity to continuity of reference: Subject pronoun use in Spanish in New York City”. En Manel Lacorte y Jennifer Leeman, Eds., Español en Estados Unidos y otros contextos: Sociolingüística, ideología y pedagogía. Madrid: Iberoamericana. 111-136.

. 2013. "Social class and gender impacting change in bilingual settings: Spanish subject pronoun use in New York". Language in Society 42.4: 429-452.

Silva-Corvalán, Carmen. 1982. "Subject expression and placement in Mexican-American Spanish". En Jon Amastae y Lucía Elías-Olivares, Eds., Spanish in the United States: Sociolinguistic aspects. Cambridge: Cambridge University Press. 93-120.

Silva-Corvalán, Carmen. 1994. Language contact and change: Spanish in Los Angeles.

Oxford: Clarendon. . 2001. Sociolingüistica y pragmática del español. Washington, D. C.: Georgetown University Press. 
Solomon, Julie. 1999. Phonological and syntactic variation in the Spanish of Valladolid, Yucatán. Tesis doctoral, Stanford University.

Tagliamonte, Sali A. 2006. Analysing sociolinguistic variation. Cambridge, NY: Cambridge University Press.

Torres Cacoullos, Rena y Catherine E. Travis. 2010. "Variable yo expression in New Mexico: English influence?”. En Susana Rivera-Mills y Daniel Villa, Eds., Spanish of the U.S. Southwest: A language in transition. Madrid: Iberoamericana/Vervuert. 185-206. . 2015. "Foundations for the study of subject pronoun expression in Spanish in contact with English: Assessing interlinguistic (dis)similarity via intralinguistic variability”. En Ana M. Carvalho, Rafael Orozco y Naomi Lapidus Shin, Eds., Subject pronoun expression in Spanish: A cross-dialectal perspective. Georgetown: Georgetown University Press. 81-100.

Travis, Catherine E. 2005. "The yo-yo effect: Priming in subject expression in Colombian Spanish”. En Randall Gess \& Edward J Rubin, Eds., Selected Papers from the 34 th Linguistic Symposium on Romance Languages (LSRL). Amsterdam: Benjamins. 329349 .

. 2007. "Genre effects on subject expression in Spanish: Priming in narrative and conversation". Language Variation and Change 19: 101-135.

Travis, Catherine E. y Rena Torres Cacoullos. 2012. "What do subject pronouns do in discourse? Cognitive, mechanical and constructional factors in variation". Cognitive Linguistics 23.4: 711-748. 
